\title{
Achados otoneurológicos em pacientes com diagnóstico de alça vascular de VIII par craniano na ressonância magnética
}

\section{Neurotology findings in patients with diagnosis of vascular loop of cranial nerves VIII in magnetic resonance imaging}

\author{
Francisco Luiz Busato Grocoske1, Rita de Cássia C. G. Mendes², Ronaldo Vosguerau³, Marcos Mocellin", \\ Maria Theresa Costa Ramos de Oliveira ${ }^{3}$, Heloisa Nardi Koerner ${ }^{3}$. \\ 1) Médico (a).Residente do $3^{\circ}$ ano do Serviço de Otorrinolaringologia do Hospital de Clínicas da Universidade Federal do Paraná \\ 2) Médica Otorrinolaringologista. Médica Responsável do Ambulatório de Otoneurologia do Serviço de Otorrinolaringologia do Hospital de Clínicas da Universidade Federal \\ do Paraná. \\ 3) Médico Radiologista.Médico do D API - Diagnostico Avancado por Imagem - Curitiba - PR. \\ 4) Doutor Em Otorrinolaringologia. Professor Titular do Serviço de Otorrinolaringologia do Hospital de Clínicas da Universidade Federal do Paraná. \\ Instituição: Hospital de Clínicas da Universidade Federal do Paraná \\ Curitiba / PR - Brasil. \\ Endereço para correspondência: Francisco L. B. Grocoske - Rua Prof. Pedro V. P. de Souza, 1541 - Apto. 221 - Curitiba / PR - Brasil - CEP: $81200-100$ - Telefax: (+55 \\ 41) 3373-1952-E-mail: francisco.grocoske@terra.com.br \\ Artigo recebido em 15 de Março de 2011. Artigo aprovado em 14 de Agosto de 2011
}

\section{RESUMO}

Introdução: A compressão vascular por vasos redundantes sobre o VIII par craniano vem sendo estudada desde a década de 80, e diversos autores propuseram correlações entre a compressão e os achados otoneurológicos (vertigem, zumbido, hipoacusia, audiometria e achados eletrofisiológicos).

Objetivo: Analisar e correlacionar os diferentes sinais e sintomas otoneurológicos, os achados audiológicos e sua incidência em indivíduos com diagnóstico de alça vascular (AV) de VIII par craniano por Ressonância Magnética (RM).

Método: Estudo retrospectivo através da análise de prontuários de 47 pacientes atendidos no ambulatório de Otoneurologia do Hospital de Clínicas da UFPR. Todos os pacientes possuíam exames de RM com imagens compatíveis com AV de VIII par craniano.

Resultados: O zumbido foi o sintoma mais frequente, em $83 \%$ dos pacientes, seguido de hipoacusia (60\%) e vertigem (36\%). A audiometria apresentava alterações em 89\%, o PEATE em $33 \%$ e o VENG em 17\% dos pacientes. Não foi encontrada relação estatisticamente significante entre o zumbido ou a hipoacusia e a presença de AV na RM. Somente 36\% dos pacientes tinham queixas de vertigem, o principal sintoma descrito na teoria da compressão vascular do VIII par. Tanto na Audiometria quanto no PEATE não foi encontrada relação estatisticamente significante entre o exame e a presença de AV na RM.

Conclusão: Os resultados mostram independência entre os achados da RM, quadro clínico e resultados audiológicos ( $p>0,05)$ sugerindo que não exista relação direta e exclusiva entre o diagnóstico de alça vascular na RM e o quadro clínico otoneurológico correspondente.

Palavras-chave: nervo vestibulococlear, zumbido, perda auditiva, audiometria, imagem por ressonância magnética.

\section{SUMMARY}

Introduction: The vascular compression by redundant vessels under the VIII cranial nerves has been studied since the 80's, and many authors proposed correlations between the compression and the otoneurological findings (vertigo, tinnitus, hypoacusis, audiometry and electrophysiological findings). Objective: Analyze and correlate the different signs and otoneurological symptoms, the audiological findings and its incidence over individuals with Vascular Loop (VL) diagnosis of VIII cranial nerves by magnetic resonance imaging (MRI). Method: Retrospective study through the analysis of medical records of 47 patients attended in the otoneurology clinic of Clinical Hospital of UFPR. All the patients have MRI exams with compatible pictures of VL of the VIII cranial nerves.

Results: The tinnitus was the most frequent symptom, in $83 \%$ of the patients, followed by hypoacusis (60\%) and vertigo (36\%). The audiometry presented alterations in $89 \%$, the brainstem evoked auditory potential in $33 \%$ and the vectoelectronystagmography in $17 \%$ of the patients. Was not found statistically significant relation between the buzz or hypoacusis, and the presence of VL in MRI. Only 36\% of patients had complaints of vertigo, the main symptom described in theory of vascular compression of the VIII pair of nerve. As in the audiometry and in brainstem evoked auditory potential was not found a statistically significant relation between the exam and the presence of the VL in the RMI.

Conclusion: The results show independence between the findings of the RMI, clinical picture and audiological results $(\mathrm{p}>0,05)$ suggesting that there are no exclusive and direct relation between the diagnosis of vascular loop in the MRI and the clinical picture matching.

Keywords: vestibulocochlear nerve, tinnitus, hearing loss, audiometry, MRI (magnetic resonance imaging). 


\section{INTRODUÇÃO}

Compressões vasculares por vasos anômalos ou redundantes sobre nervos cranianos, e o estabelecimento de uma correlação estatisticamente relevante entre a compressão e os sintomas neurológicos vêm sendo estudadas por diversos autores desde a década de 30.

A alça vascular causando compressão do $5^{\circ}$ nervo craniano resultando em neuralgia do trigêmeo foi sugerida primeiramente por DANDY em 1934 (1), e mais tarde, por Gardner e Miklos (2) em 1959. Este conceito foi então extensamente ampliado para tentar explicar desordens de vários nervos cranianos. Estas apresentações são conhecidas como síndromes compressivas, que incluem espasmo hemifacial, neuralgia glossofaríngea, neuralgia geniculada, e, mais recentemente, uma síndrome de compressão do nervo vestíbulo coclear. Embora McKENZIE (3) sugeriu em 1936 que a Doença de Ménière poderia ser causada por uma relação anormal da artéria cerebelar anterior inferior (AICA em inglês) e o $8^{\circ}$ nervo craniano, este conceito não foi reintroduzido até 1975, quando JANETTA (4) propôs então que alças arteriais redundantes no ângulo ponto cerebelar (APC) poderiam interferir com o oitavo par craniano resultando em sintomas de vertigem grave, zumbido e perda auditiva sensório neural.

Todas as teorias fisiopatológicas baseiam-se em um tema comum: um vaso redundante aderindo a um nervo craniano causando excitação crônica ectópica. Isto resulta em uma reorganização do núcleo nervoso causando hiperfunção nervosa. Foi sugerido que este processo patológico seria restrito à zona de entrada da raiz do nervo craniano, como esta sendo a área juncional entre a mielina central e a periférica, onde defeitos poderiam ocorrer. LANG mostrou que o segmento central do 8 o par craniano tem um comprimento de $10 \mathrm{~mm}$ (6 a $15 \mathrm{~mm}$ ) e um comprimento intracisternal de $15 \mathrm{~mm}$ (8.5 a $22 \mathrm{~mm}$ ). Ele achou a zona de transição do oitavo nervo craniano variando do tronco cerebral ao fundo do Conduto Auditivo Interno (CAI). A histologia conhecida seria compatível com a ocorrência de uma síndrome de compressão neurovascular do oitavo nervo craniano, em qualquer lugar do trajeto, desde o tronco cerebral até o CAI.

A existência das Síndromes de compressão vascular (SCV), e especialmente aqui a compressão do VIII par, foi inicialmente inferida por analogia à síndrome do espasmo hemifacial, na qual é bem aceito que a causa é um vaso aberrante comprimindo o nervo. JannetTa e Moller (5), de Pittsburgh, EUA, foram os pioneiros no diagnóstico e tratamento desta enfermidade. Porém nas outras síndromes de compressão vascular propostas pelos mesmos autores (neuralgia trigeminal, glossofaríngea, etc.) não foram tão relacionadas e aceitas como consequência da compressão vascular.

Os mesmos autores, em 1984, descreveram então uma SCV relacionada ao $8^{\circ}$ par, chamada então de vertigem posicional incapacitante ("disabling positional vertigo"), caracterizada por história de: vertigem, geralmente em crises de duração curta (2-3min); hipoacusia, de grau leve a moderado, tipicamente com um entalhe em frequências medias na audiometria e uma latência entre ondas I-III aumentada no potencial evocado auditivo de tronco encefálico (PEATE).

A denominação "vertigem posicional" parece ser inapropriada, já que autores subsequentes não enfatizaram este achado, como foi no caso de nosso estudo, como veremos na discussão.

JANETTA e cols. propuseram como tratamento à colocação de material absorvível entre o vaso e o nervo. Embora o objetivo fosse separar o efeito do vaso sobre o nervo, outros autores $(6,7)$ questionaram se este procedimento simplesmente danificaria a estrutura do VIII par, ou ainda seria totalmente infundada. Outros (8) sugeriram a completa secção do nervo como tratamento. Há também propostas de tratamento clínico, com uso de carbamazepina, com bons resultados.

Assim, apesar nos inúmeros artigos publicados desta condição, a simples existência das SCV continua a ser questionada. Os sintomas da SCV são todos inespecíficos, podendo ser facilmente encontrados em outras condições como doença de Ménière, enxaqueca ou simplesmente uma intolerância inata ao movimento. Não há ainda um teste específico convincente. Os achados de PEATE propostos por Moller (9) como característicos de SCV não seriam considerados por muitos autores, COMO SCHWABER e Hall (6,7). Ainda, segundo Parnes (10), nenhum achado de tomografia axial computadorizada (TAC) ou ressonância magnética (RM) relacionado com vasos sanguíneos seria diagnóstico, já que vasos cruzando o VII par são encontrados em ao menos $1 / 3$ de indivíduos assintomáticos.

A variabilidade da anatomia vascular do APC e do canal auditivo interno (CAI) em pessoas normais contribuiu para com a controvérsia em torno do conceito das síndromes de compressão vascular. Tanto estudos post-mortem como radiológicos investigaram o local da alça vascular formada pela AICA no APC $(12,13,14)$. Os resultados mostraram discrepâncias consideráveis, embora todos os estudos indicassem que alças vasculares entrariam no CAI em uma minoria dos pacientes. 
Em essência, a SCV do VIII par seria uma síndrome de sintomas vestibulares (vertigens rápidas, combinadas com intolerância ao movimento) e audiológicos que respondem ao tratamento com medicações para neuralgia (carbamazepina) e onde outras causas semelhantes (Ménière, enxaqueca) tenham sido afastadas.

Logicamente, nenhum dos sintomas desta síndrome é exclusivo de uma origem vascular, mas as suas características mais específicas (sintomas intensos e de curta duração, que respondem a medicação para neuralgia) demonstram um problema elétrico, pois qualquer alteração que irrite o nervo vestibular irá causar sintomas semelhantes, como já foi demonstrado na literatura por Moon e HaIN (11).

Assim, uma denominação mais razoável para esta síndrome é Paroxismia Vestibular (PV).

Há evidências de que a compressão vascular do VIII nervo craniano seja a causa mais provável da paroxismia vestibular $(5,12)$, com hiperatividade e progressiva perda funcional. Pode ser diagnosticada pela ocorrência de episódios curtos de tontura rotatória (vertigem), precipitados ou modulados pela mudança da posição da cabeça, podendo estar associada à hipoacusia e/ou zumbidos (13).

No diagnóstico da PV, é importante diferenciá-la de outras síndromes que cursam com vertigem episódica de curta duração, como VPPB, doença de Ménière e neurite vestibular. A vertigem da PV desencadeada por movimentos da cabeça não é fatigável como nos pacientes com VPPB, e seus sintomas não são aliviados com uso de supressores vestibulares (5). Na PV os pacientes não têm a perda auditiva flutuante característica da doença de Menière.

Tanto a Tomografia Axial Computadorizada (TAC) como a Ressonância Magnética (RNM) com achados de alça vascular ainda não se mostraram específicas como auxílio diagnóstico, porém novos tipos de RNM, como FT-FISS podem ser mais específicos. Recentemente, McDermotT et al. (14), em um estudo extenso, propôs que alças que se estendem para dentro do CAI são mais significativas como fonte de sintomas auditivos unilaterais, como zumbido, do que aquelas acerca do APC. Um PEATEanormal do lado dos sintomas suporta o diagnóstico, mas um exame normal não o exclui. Desta forma, o diagnóstico definitivo somente é conseguido após exploração cirúrgica, com cura no followup. Obviamente, extrema cautela deve ser empregada na indicação cirúrgica, pois é necessário um acesso neurocirúrgico a o tronco cerebral, devendo ser considerado em último caso em falha de resposta a todas as outras alternativas clínicas.

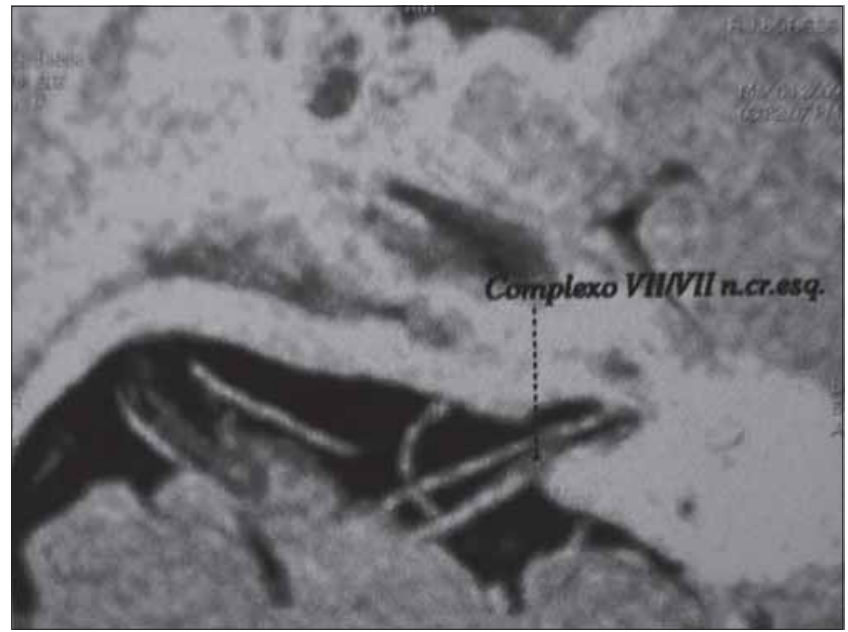

Figura 1. Alça Vascular de VIII par craniano.

O objetido deste artigo é analisar e correlacionar os sinais e sintomas otoneurológicos, os achados audiológicos e sua incidência em indivíduos com alça vascular (AV) de VII/VIII par demonstrado por imagens de ressonância nuclear magnética de ângulo ponto cerebelar.

\section{MÉTODO}

Foram analisados 47 pacientes atendidos no ambulatório de Otoneurologia do Hospital de Clínicas da Universidade Federal do Paraná, no período de Junho de 2009 a Maio de 2010.

Todos os pacientes tinham uma ou mais queixas otoneurológicas de perda de audição, vertigem e zumbido. Foram submetidos aos seguintes exames complementares: Audiometria, Vectoeletronistagmografia e PEATE, e todos possuíam exames de Ressonância Magnética com imagens compatíveis com Alça Vascular de VIII par craniano (Figura 1). Neste exame usamos a classificação proposta por McDeRmotт et al. (14) em relação ao local da alça no trajeto do nervo:

Grau I: Alça vascular totalmente fora do Conduto Auditivo Interno

Grau II: Alça vascular insinuando-se em até 50\% no Conduto Auditivo Interno

Grau III: Alça vascular insinuando-se mais de 50\% no Conduto Auditivo Interno

Procedeu-se ao tratamento estatístico julgado adequado de acordo com a natureza dos dados coletados, que consistiu na elaboração de tabelas e gráficos para o estudo 
de frequências das variáveis isoladamente, bem como tabelas de dupla entrada para distribuição e análise estatística da variável "alça vascular" por "zumbido", "hipoacusia", "audiometria" e "peate". Na análise estatística foram utilizados os testes de qui-quadrado e Fisher, levando-se em conta as frequências esperadas, com nível de significância $\mathrm{p}<0,05$.

Este trabalho foi aprovado pelo comitê de ética do HC/UFPR sob o numero 2248.142/2010-06.

Tabela I. Distribuição da amostra por sexo.

\begin{tabular}{lcc}
\hline Sexo & $N$ & $\%$ \\
\hline Masculino & 24 & $51,1 \%$ \\
Feminino & 23 & $48,9 \%$ \\
\hline Total & 47 & $100 \%$ \\
\hline
\end{tabular}

Tabela 2. Distribuição do sintoma de zumbido.

\begin{tabular}{lcc}
\hline Lado & $N$ & $\%$ \\
\hline Ouvido Direito & 6 & $12,8 \%$ \\
Ouvido Esquerdo & 14 & $29,8 \%$ \\
Bilateral & 19 & $40,4 \%$ \\
SemZumbido & 8 & $17,0 \%$ \\
\hline Total & 47 & $100 \%$ \\
\hline
\end{tabular}

Tabela 3. Distribuição do sintoma de hipoacusia.

\begin{tabular}{lcc}
\hline Lado & $N$ & $\%$ \\
\hline OuvidoDireito & 10 & $21,3 \%$ \\
Ouvido Esquerdo & 5 & $10,6 \%$ \\
Bilateral & 13 & $27,7 \%$ \\
SemHipoacusia & 19 & $40,4 \%$ \\
\hline Total & 47 & $100 \%$ \\
\hline
\end{tabular}

Tabela 4. Distribuição do sintoma de vertigem.

\begin{tabular}{lcc}
\hline Vertigem & $N$ & $\%$ \\
\hline ComVertigem & 17 & $36,2 \%$ \\
SemVertigem & 30 & $63,8 \%$ \\
\hline Total & 47 & $100 \%$
\end{tabular}

Tabela 5. Distribuição da amostra em relação a audiometria.

\begin{tabular}{lcc}
\hline Lado Alterado & $N$ & $\%$ \\
\hline Ouvido Direito & 7 & $14,9 \%$ \\
Ouvido Esquerdo & 6 & $12,8 \%$ \\
Bilateral & 29 & $61,7 \%$ \\
Audiometria Normal & 5 & $10,6 \%$ \\
\hline Total & 47 & $100 \%$ \\
\hline
\end{tabular}

\section{RESULTADOS}

Conforme a Tabela 1, os pacientes tinham idade média de 56,1 anos, variando entre 23 e 78 anos, distribuídos equilibradamente entre os sexos.

O Gráfico 1 e as Tabelas 2, 3, 4, 5, 6, 7, 8 e 9 apresentam as frequências dos sintomas otneurologicos e exames alterados dos pacientes do estudo.

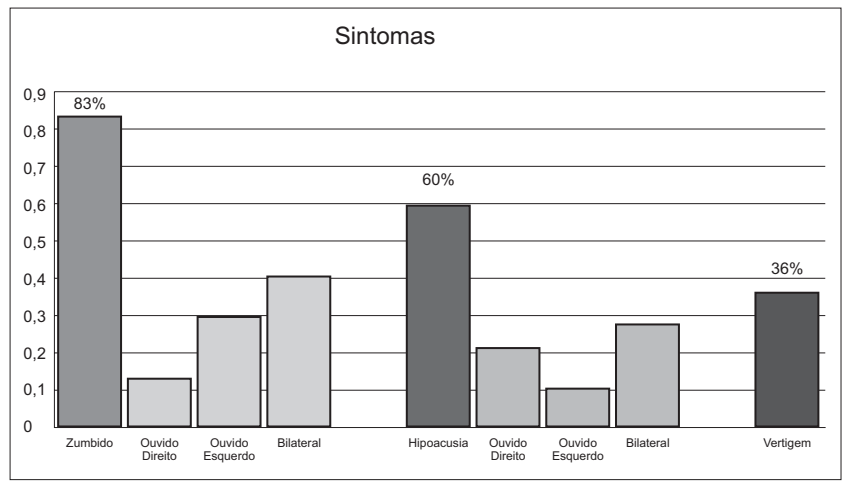

Gráfico 1. Distribuição dos sintoma.

Tabela 6. Distribuição da amostra em relação ao Peate.

\begin{tabular}{lcc}
\hline Lado Alterado & $N$ & $\%$ \\
\hline Ouvido Direito & 3 & $6,4 \%$ \\
Ouvido Esquerdo & 5 & $10,6 \%$ \\
Bilateral & 8 & $17,0 \%$ \\
Peate Normal & 31 & $66,0 \%$ \\
\hline Total & 47 & $100 \%$ \\
\hline
\end{tabular}

Tabela 7. Distribuição da amostra em relação ao Veng.

\begin{tabular}{lcc}
\hline Tipo DeAlteração & $N$ & $\%$ \\
\hline Deficitária & 3 & $6,4 \%$ \\
Irritativa & 5 & $10,6 \%$ \\
Veng Normal & 39 & $83,0 \%$ \\
\hline Total & 47 & $100 \%$ \\
\hline
\end{tabular}

Tabela 8. Distribuição da amostra em relação ao lado afetado naRMn.

\begin{tabular}{lcc}
\hline Lado & $N$ & $\%$ \\
\hline Ouvido Direito & 13 & $27,7 \%$ \\
Ouvido Esquerdo & 9 & $19,1 \%$ \\
Bilateral & 25 & $53,2 \%$ \\
\hline Total & 47 & $100 \%$ \\
\hline
\end{tabular}


Tabela 9. Distribuição da amostra em relação ao tipo da alça vascular.

\begin{tabular}{lcc}
\hline Tipo daAlça Vascular & $N$ & $\%$ \\
\hline Tipo I & 15 & $31,9 \%$ \\
Tipo II & 17 & $36,2 \%$ \\
Tipo III & 6 & $12,8 \%$ \\
Não Classificado & 9 & $19,1 \%$ \\
\hline Total & 47 & $100 \%$ \\
\hline
\end{tabular}

Vimos que, em relaçãoaos sintomas otoneurológicos, o zumbido foi o mais frequente, sendo presente em 39 (83\%) dos pacientes, seguido de hipoacusia, em 28 (59,6\%) e vertigem, em 17 (36,2\%) dos pacientes.

O zumbido foi presente em 39 (83\%) dos pacientes, mais comum bilateralmente. Oito pacientes (17\%) não tinham queixa de zumbido.

A hipoacusia foi um sintoma presente em 28 (59,6\%) pacientes, com os restantes $19(40,4 \%)$ sem esta queixa.

A vertigem foi presente em $17(36,2 \%)$ dos pacientes, com $30(63,8 \%)$ sem esta queixa.

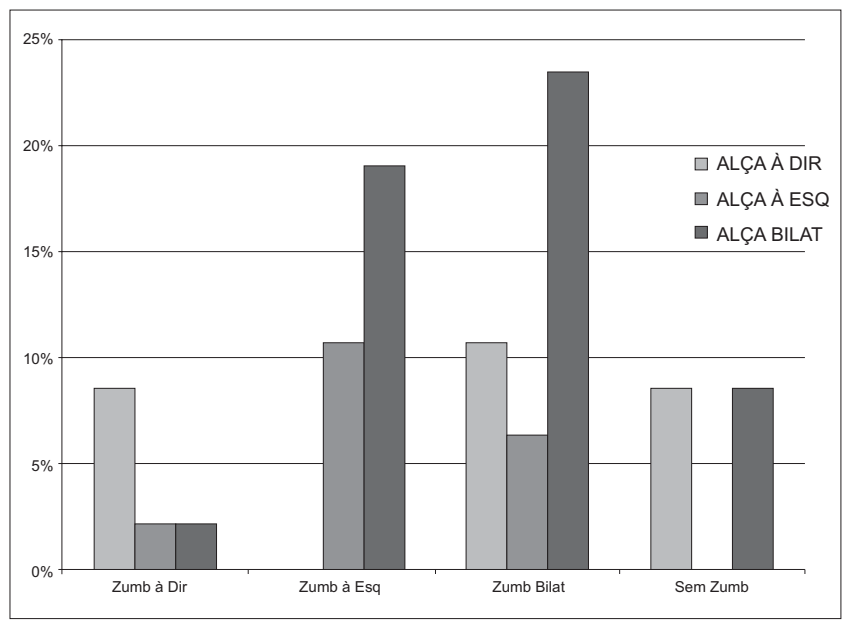

Grafico 2. Distribuição do sintoma de zumbido.

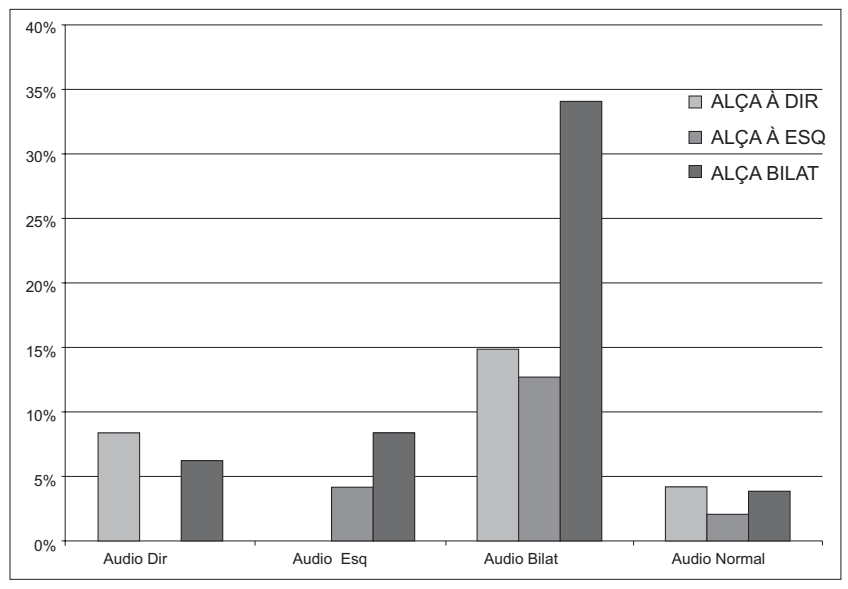

Grafico 4. Distribuição dos resultados audiométricos.
A audiometria a presentava alterações em 89,4\% dos pacientes, sendo mais frequentes as perdas bilaterais. Cinco pacientes (10,6\%) tinham audiometria normal.

Trinta e um pacientes (66\%) tinham PEATE normal, enquanto que 16 (33\%) tinham este exame alterado.

Dos 47 pacientes do estudo, 8 (17\%) apresentavam VENG alterado.

Todos os pacientes tinham exame de RMN alterada, com diagnóstico de alça vascular de VIII par craniano. Vinte e cinco deles $(53,2 \%)$ eram bilaterais, o restante $(46,8 \%)$ era unilateral.

Quanto ao tipo de alça vascular na RM, 9 pacientes (19,1\%) não a tinham classificada, enquanto que $15(31,9 \%)$ possuíam o tipo I, 17 (36,2\%) o tipo II e 16 (12,8\%) o tipo III.

Procedemos então ao cruzamento dos dados, analisando os achados de RNM versus os sintomas otoneurológicos e os resultados dos exames complementares, conforme apresentamos nos Gráficos 2, 3, 4 e 5 a nas Tabelas 10,11, $12,13,14,15,16,17,18$ e 19 .

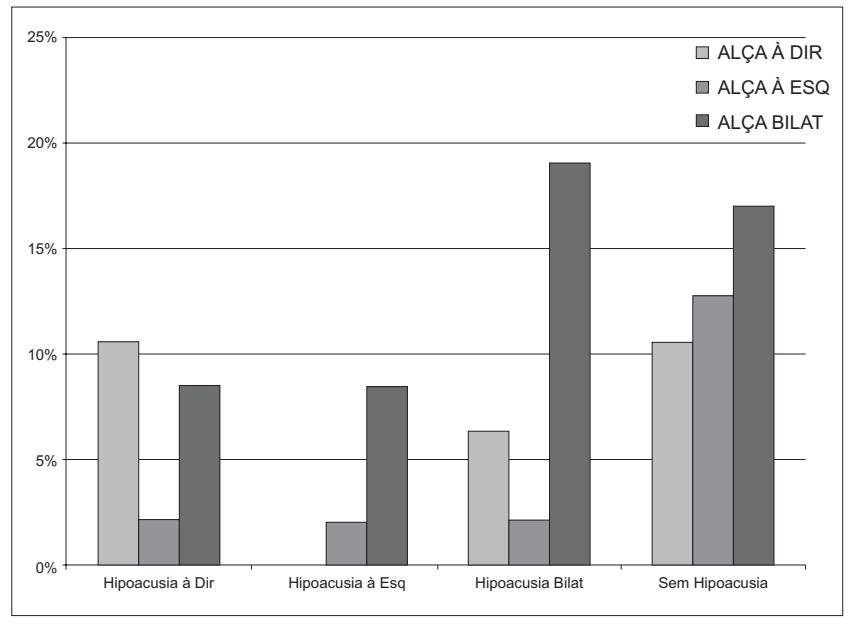

Grafico 3. Distribuição do sintoma de hipoacusia.

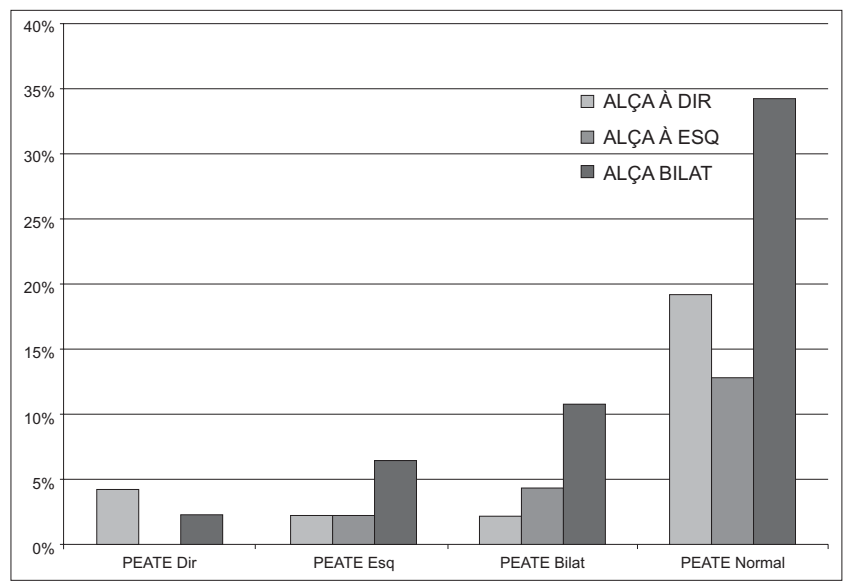

Grafíco 5. Distribuição dos resultados do PEATE. 
Tabela I0. Distribuição da alça por zumbido em 47 pacientes.

\begin{tabular}{|c|c|c|c|c|c|c|c|c|c|c|}
\hline \multirow[b]{2}{*}{ Alça } & \multicolumn{6}{|c|}{ Zumbido/Ouvido } & \multirow{2}{*}{$\begin{array}{l}\text { Sem } \\
\text { Zumb }\end{array}$} & \multirow[t]{2}{*}{$\%$} & \multirow[t]{2}{*}{ Total } & \multirow[t]{2}{*}{$\%$} \\
\hline & Direito & $\%$ & Esquerdo & $\%$ & Bilateral & $\%$ & & & & \\
\hline ADireita & 4 & 8,51 & 0 & 0,00 & 5 & 10,64 & 4 & 8,51 & 13 & 27,66 \\
\hline AEsquerda & 1 & 2,13 & 5 & 10,64 & 3 & 6,38 & 0 & 0,00 & 9 & 19,15 \\
\hline Bilateral & 1 & 2,13 & 9 & 19,15 & 11 & 23,40 & 4 & 8,51 & 25 & 53,19 \\
\hline Total & 6 & 12,77 & 14 & 29,79 & 19 & 40,43 & 8 & 17,02 & 47 & 100,00 \\
\hline
\end{tabular}

Tabela II I Análise estatística da frequência da alça por lado percebido do zumbido em 39 pacientes.

\begin{tabular}{lcccc}
\hline $\begin{array}{l}\text { Zumbido } \\
\text { Alça }\end{array}$ & \multicolumn{2}{c}{ Ouvido } & Total & Pireito ou \\
Esquerdo & Bilateral & & \\
\hline Direita ou Esquerda & 10 & 8 & 18 & 0,62 \\
Bilateral & 10 & 11 & 21 & \\
\hline Total & 20 & 19 & 39 & \\
\hline
\end{tabular}

Tabela I2. Análise estatística da frequência da alça por presença de zumbido em 47 pacientes.

\begin{tabular}{lcccc}
\hline $\begin{array}{l}\text { Zumbido } \\
\text { Alça }\end{array}$ & \multicolumn{2}{c}{ Presença } & Total & $P$ \\
\hline Direita Ou Esquerda & 18 & 4 & 22 & 0,57 \\
Bilateral & 21 & 4 & 25 & \\
\hline Total & 39 & 8 & 47 & \\
\hline
\end{tabular}

Tabela I 3. Distribuição da alça por hipoacusia em 47 pacientes.

\begin{tabular}{|c|c|c|c|c|c|c|c|c|c|c|}
\hline \multirow{2}{*}{$\begin{array}{l}\text { Hipoacusia } \\
\text { Alça }\end{array}$} & \multicolumn{6}{|c|}{ Ouvido } & \multirow[t]{2}{*}{ Sem } & \multirow[t]{2}{*}{$\%$} & \multirow{2}{*}{ Total } & \multirow[t]{2}{*}{$\%$} \\
\hline & Direito & $\%$ & Esquerdo & $\%$ & Bilateral & $\%$ & & & & \\
\hline ADireita & 5 & 10,64 & 0 & 0,00 & 3 & 6,38 & 5 & 10,64 & 13 & 27,66 \\
\hline AEsquerda & I & 2,13 & I & 2,13 & I & 2,13 & 6 & 12,76 & 9 & 19,15 \\
\hline Bilateral & 4 & 8,51 & 4 & 8,51 & 9 & 19,15 & 8 & 17,02 & 25 & 53,19 \\
\hline Total & 10 & 21,28 & 5 & 10,64 & 13 & 27,66 & 19 & 40,42 & 47 & 100,00 \\
\hline
\end{tabular}

Tabela |4. Análise estatística da frequência da alça por lado percebido da hipoacusia em 28 pacientes.

\begin{tabular}{lcccc}
\hline Hipoacusia & \multicolumn{2}{c}{ Ouvido } & Total & $P$ \\
Alça & $\begin{array}{c}\text { Direito ou } \\
\text { Esquerdo }\end{array}$ & Bilateral & & \\
\hline Direita ou Esquerda & 7 & 4 & 11 & 0,39 \\
Bilateral & 8 & 9 & 17 & \\
\hline Total & 15 & 13 & 28 &
\end{tabular}

Tabela | 6. Distribuição da alça por audiometria em 47 pacientes.

\begin{tabular}{|c|c|c|c|c|c|c|c|c|c|c|}
\hline \multirow{2}{*}{$\begin{array}{l}\text { Audiometria } \\
\text { Alça }\end{array}$} & \multicolumn{6}{|c|}{ Ouvido } & \multirow[t]{2}{*}{ Sem } & \multirow[t]{2}{*}{$\%$} & \multirow[t]{2}{*}{ Total } & \multirow[t]{2}{*}{$\%$} \\
\hline & Direito & $\%$ & Esquerdo & $\%$ & Bilateral & $\%$ & & & & \\
\hline ADireita & 4 & 8,51 & 0 & 0,00 & 7 & | 4,89 & 2 & 4,26 & 13 & 27,66 \\
\hline AEsquerda & 0 & 0,00 & 2 & 4,26 & 6 & 12,77 & I & 2,12 & 9 & 19,15 \\
\hline Bilateral & 3 & 6,38 & 4 & 8,51 & 16 & 34,04 & 2 & 4,26 & 25 & 53,19 \\
\hline Total & 7 & 14,89 & 6 & 12,77 & 29 & 61,70 & 5 & 10,64 & 47 & 100,00 \\
\hline
\end{tabular}

Tabela 17. Análise estatística da frequência da alça por audiometria em 42 pacientes.

\begin{tabular}{|c|c|c|c|c|}
\hline \multirow{2}{*}{$\begin{array}{l}\text { Audiometria } \\
\text { Alça }\end{array}$} & \multicolumn{2}{|c|}{ Ouvido } & \multirow{2}{*}{ Total } & \multirow[t]{2}{*}{$P$} \\
\hline & $\begin{array}{l}\text { Direito ou } \\
\text { Esquerdo }\end{array}$ & Bilateral & & \\
\hline Direita ou Esquerda & 6 & 13 & 19 & 0,80 \\
\hline Bilateral & 7 & 16 & 23 & \\
\hline Total & 13 & 29 & 42 & \\
\hline
\end{tabular}


Tabela | 8. Distribuição da alça por peate em 47 pacientes.

\begin{tabular}{|c|c|c|c|c|c|c|c|c|c|c|}
\hline \multirow{2}{*}{$\begin{array}{l}\text { PEATE } \\
\text { Alça }\end{array}$} & \multicolumn{6}{|c|}{ Ouvido } & \multirow[t]{2}{*}{ Sem } & \multirow[t]{2}{*}{$\%$} & \multirow[t]{2}{*}{ Total } & \multirow[t]{2}{*}{$\%$} \\
\hline & Direito & $\%$ & Esquerdo & $\%$ & Bilateral & $\%$ & & & & \\
\hline ADireita & 2 & 4,26 & I & 2,13 & I & 2,13 & 9 & 19,15 & 13 & 27,66 \\
\hline AEsquerda & 0 & 0,00 & I & 2,13 & 2 & 4,26 & 6 & 12,77 & 9 & 19,15 \\
\hline Bilateral & I & 2,13 & 3 & 6,38 & 5 & 10,64 & 16 & 34,04 & 25 & 53,19 \\
\hline Total & 3 & 6,38 & 5 & 10,64 & 8 & 17,02 & 31 & 65,96 & 47 & 100,00 \\
\hline
\end{tabular}

Tabela | 9. Análise estatística da frequência da alça por Peate em 16 pacientes.

\begin{tabular}{|c|c|c|c|c|}
\hline \multirow{2}{*}{$\begin{array}{l}\text { Audiometria } \\
\text { Alça }\end{array}$} & \multicolumn{2}{|c|}{ Ouvido } & \multirow[t]{2}{*}{ Total } & \multirow[t]{2}{*}{$P$} \\
\hline & $\begin{array}{l}\text { Direito ou } \\
\text { Esquerdo }\end{array}$ & Bilateral & & \\
\hline Direita ou Esquerda & 4 & 3 & 7 & 0,50 \\
\hline Bilateral & 4 & 5 & 9 & \\
\hline Total & 8 & 8 & 16 & \\
\hline
\end{tabular}

Dos pacientes com queixa de zumbido à direita, 4 $(8,51 \%)$ possuíam alça vascular correspondente na RM. À esquerda, 5 pacientes (10,6\%) tinham zumbido e alça do mesmo lado, e dos 19 pacientes com queixa bilateral, 11 $(23,4 \%)$ tinham imagem na RM de alça vascular bilateral. Oito pacientes (17\%) do estudo não tinham queixa de zumbido e apresentavam alça vascular na RM. Existe independência entre a localização da alça vascular e o lado percebido do zumbido. Também existe independência entre a localização da alça vascular e a simples presença do zumbido, independentemente do lado percebido, assim como do tipo percebido do zumbido, pulsátil ou não.

Relacionando-se a queixa de hipoacusia e lado da alça, cinco dos treze pacientes com alça vascular à direita possuíam hipoacusia do mesmo lado, somente um paciente dos nove com alça vascular à esquerda referia hipoacusia também à esquerda e nove dos vinte e cinco com alça bilateral também referiam hipoacusia bilateral. Existe independência tanto entre a localização da alça vascular e o lado percebido de hipoacusia como entre a presença da alça vascular e a sintomatologia de hipoacusia, independentemente do lado percebido.

Em relaçãoaos resultados audiométricos, quatro dos treze pacientes com alça vascular à direita possuíam audiometria alterada do mesmo lado, dois pacientes dos nove com alça vascular à esquerda possuíam audiometria alterada à esquerda e dezesseis dos vinte e cinco com alça bilateral também possuíam audiometria alterada bilateralmente. Existe independência entre a localização da alça vascular e o lado alterado da audiometria.

Analisando os resultados do PEATE, dois dos treze pacientes com alça vascular à direita possuíam PEATE alterado do mesmo lado, somente um paciente dos nove com alça vascular à esquerda possuía PEATE alterado à esquerda e cinco dos vinte e cinco com alça bilateral também possuíam PEATE alterado bilateralmente.

Existe independência entre a localização da alça vascular e o lado alterado do PEATE.

\section{DISCUSSÃO}

Observamos Que Nos Sintomas apresentados pelos pacientes, tanto em relação ao zumbido quanto a hipoacusia, não há significância estatística ente o sintoma e à presença de alça vascular na RNM, seja em relação ao lado percebido, ou mesmo pela simples presença do sintoma. No zumbido, mesmo isolando-se somente os casos do tipo pulsátil, teoricamente o que mais se relacionaria à alça vascular, notamos que a independência se mantém, não havendo relação direta entre alça e zumbido. Isto também é reforçado pela pequena quantidade de pacientes do estudo ( 7 , ou 14,8\%), com queixa de zumbido do tipo pulsátil

Estes achados corroboram aqueles de Makins (15), que não viu diferença significativa em relação à presença de alça vascular, entre orelhas com sinais e sintomas e orelhas sadias, sugerindo que a presença de alça vascular na RNM não é patológico, mas um achado de exame.

Esta independência manteve-se na análise dos exames audiológicos (Audiometria e PEATE), onde a presença da alça vascular na RNM também não apresentou uma significância estatística com os dados dos exames audiológicos. Isto reforça os achados sintomatológicos, neste caso sem a subjetividade inerente dos sintomas.

Observamos também uma incidência relativamente baixa de PEATE alterado, em 34\% dos pacientes, já que pela teoria da compressão vascular, esperava-se uma alteração mais frequente na transmissão do estímulo, representada por um PEATE alterado.

Também citamos a incidência reduzida do sintoma da vertigem, (36,2\% dos pacientes), já que este foi o 
principal sintoma descrito nos primeiros estudos e na teoria da compressão vascular do VIII par.

\section{CONCLUSÃO}

A presença do conflito neurovascular do VII/VIII par craniano nas imagens de RNM não justificou por si só os sinais e sintomas otoneurológicos e as alterações audiovestibulares encontradas nos indivíduos avaliados neste estudo.

\section{REFERÊNCIAS BIBLIOGRÁFICAS}

1. Dandy WE. Concerning the cause of trigeminal neuralgia. Am J Surg. 1934, 24:447-55.

2. Gardner WJ, Miklos MV. Response of trigeminal neuralgia to decompression of sensory root: discussion of cause of trigeminal neuralgia. JAMA. 1959, 170:1773-6.

3. McKenzie KG. Intracranial division of the vestibular portion of the auditory nerve for Meniere's disease. Can Med Assoc J. 1936, 34(4):369-81.

4. Janetta PJ. Neurovascular cross-compression in patients with hyperactive dysfunction of the eighth cranial nerve. Surg Forum. 1975, 26:467-9.

5. Janetta PJ, Moller MB, Moller AR. Disabling positional vertigo. NEJM. 1984, 310:1700-1705.

6. Schwaber MK and Hall JW. Cochleovestibular nerve compression syndrome. I. Clinical features and audiovestibular findings. Laryngoscope. 1992, 102(9):1020-9.
7. Schwaber MK and Whetsell WO. Cochleovestibular nerve compression syndrome. II. Vestibular nerve histopathology and theory of pathophysiology. Laryngoscope. 1992, 102(9):1030-6.

8. McCabe BF, Harker LA. Vascular loop as a cause of vertigo. Ann ORL. 1983, 92:542-543.

9. Moller MB. Vascular compression of the eighth cranial nerve as a cause of vertigo. Keio J Med. 1991, 40(3):146150 .

10. Parnes LS, Shimotakahara SG, Pelz D, et al. Vascular relationships of the vestibulocochlear nerve on magnetic resonance imaging. Am J Otol. 1990, 11:278-281.

11. Moon I, Hain TC DelayedQuick Spins following Vestibular Nerve Section Respond to Anticonvulsant Medication. Otol Neurotol. 2005, 26:82-85.

12. Moller MB, Moller AR, Jannetta PJ, Sekhar L. Diagnosis and surgical treatment of disabling positional vertigo. $\mathrm{J}$ Neurosurg. 1986, 64:21-28.

13. Brandt T. Vertigo, its multisensory syndromes, $2^{\text {nd }} \mathrm{Ed}$. London: Springer Verlag, 1999.

14. McDermott AL, Dutt SN, Irving RM, Pahor AL, Chavda SV. Anterior inferior cerebellar artery syndrome: fact or fiction. Clin Otolaryngol. 2003, 28(2):75-80.

15. Makins A, Nikolopoulos TP, Ludman C, O'Donoghue G. Is there a Correlation Between Vascular Loops and Unilateral Auditory Symptoms? The Laryngoscope. 1998: 1739-1742. 